\title{
Adult Posterior Cranial Fossa Arachnoid Cyst: A Case Report
}

\author{
Ibrahima Berete ${ }^{*}$, Alpha Boubacar Bah'1, Hammas Nawal2 ${ }^{2}$, Mohammed Benzagmout ${ }^{3}$, \\ Khalid Chakour ${ }^{3}$, Faiz Chaoui ${ }^{3}$
}

${ }^{1}$ Department of Neurosurgery, CHU de Donka, Conakry, Guinea

${ }^{2}$ Department of Pathology, Hassan II Teaching Hospial, Fez, Morocco

${ }^{3}$ Department of Neurosurgery, Hassan II Teaching Hospital, Fez, Morocco

Email: *iberete902@icloud.com

How to cite this paper: Berete, I., Bah, A.B., Nawal, H., Benzagmout, M., Chakour, K. and Chaoui, F. (2019) Adult Posterior Cranial Fossa Arachnoid Cyst: A Case Report. Open Journal of Modern Neurosurgery, 9, 436-440.

https://doi.org/10.4236/ojmn.2019.94041

Received: March 27, 2019

Accepted: October 15, 2019

Published: October 18, 2019

Copyright (c) 2019 by author(s) and Scientific Research Publishing Inc. This work is licensed under the Creative Commons Attribution International License (CC BY 4.0).

http://creativecommons.org/licenses/by/4.0/

\begin{abstract}
Adult posterior fossa arachnoid cysts are rare lesions that are considered to be mostly congenital in origin. We present 49 -year-old man admitted with a chief complaint of tremor and balance problems for the past 2 months. $\mathrm{He}$ had ataxia on examination. Magnetic resonance imaging revealed a well circumscribed midline cystic lesion of the posterior fossa sharing the same signal characteristics as cerebrospinal fluid and non-communicating to the fourth ventricle. He underwent surgical treatment for his symptomatic posterior fossa arachnoid cyst with a good outcome at 2 years follow up.
\end{abstract}

\section{Keywords}

Posterior Fossa, Arachnoid Cyst, Subocciptal Craniotomy, Cerebellar Ataxia

\section{Introduction}

Arachnoid cysts (AC) are developmental collections of cerebrospinal-like fluid covered by arachnoid epithelium. They are believed to result from congenital malformations that can change during postnatal life. Most arachnoid cysts are located in the middle cranial fossa [1]; localizations in the posterior fossa are uncommon. The cerebello-pontine angle, the cerebellum, and the fourth ventricle are usually involved [1] [2]. AC involving the posterior fossa may be asymptomatic or may produce a wide variety of posterior fossa symptoms with little specificity such as headache, ataxia, dizziness, tinnitus, and hearing loss [2]. Different methods of surgical treatment have been reported including cystoperitoneal shunting, cyst fenestration and radical resection [3].

Herein, we report a clinical case of AC in the posterior cerebral fossa, and 
discuss the clinical features, radiological findings, therapeutic options, and differential diagnosis from other posterior fossa cysts.

\section{Case Report}

A 49-year-old man was evaluated for 2-month history of progressively worsening tremor. He was otherwise healthy, although he felt his balance was not quite as good as it used to be. He also presented chronic headache and vertigo. On examination, he had tandem gait disturbance, limb ataxia and intentional tremor. Ocular fundus exam revealed bilateral papilledema. Cranial nerves were intact, and ear, nose and throat examination were performed and weber's and Rinne's test were unremarkable.

MRI revealed slight dilation of the lateral and third ventricles and a non-enhancing well demarcated midline cyst sharing the same signal characteristics as cerebrospinal fluid, especially in diffusion imaging sequences. The cyst was non-communicating with the fourth ventricle (Figure 1).

A posterior approach was done via a midline suboccipital craniotomy in a patient installed in prone position. We opened the Dura, and discovered a translucent, smooth thin-walled cyst (Figure 2). Both the outer and inner membrane were easily dissected and completely excised. The fourth ventricle and the cisterna magna were opened and cerebrospinal fluid (CSF) flow between the fourth ventricle and subarachnoid spaces was restored. Histological examination of the

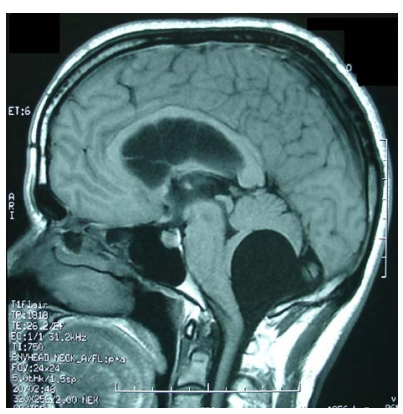

(a)

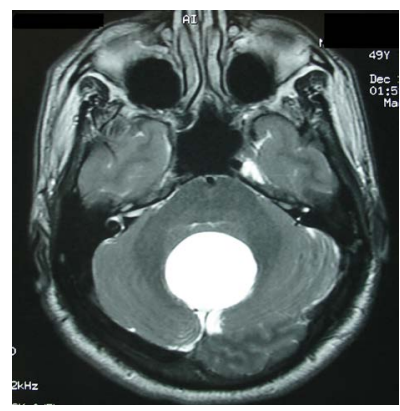

(b)

Figure 1. MRI revealed slight dilation of the lateral and third ventricles with a nonenhancing well demarcated midline cyst having the same characteristics of signal as cerebrospinal fluid in diffusion imaging sequences. The cyst was non-communicating with the fourth ventricle.

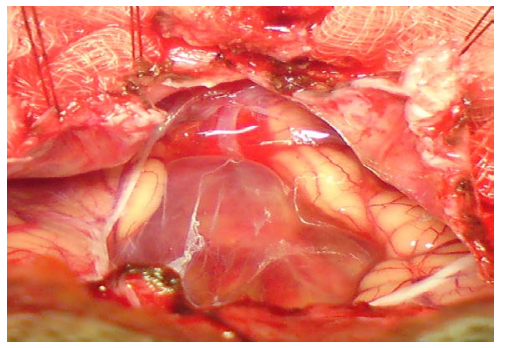

Figure 2. A posterior approach was done via a midline suboccipital craniectomy in a patient installed in prone position. The cyst wall was translucent, smooth and thin. 


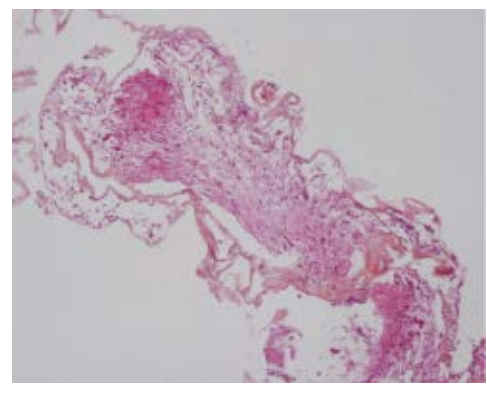

(a)

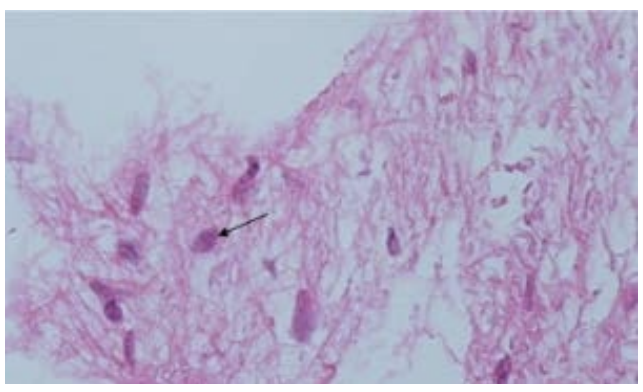

(b)

Figure 3. Thin cyst wall (a) with meningothelial cell on higher magnification (b).

excised cyst wall confirmed the diagnosis of AC (Figure 3).

Six weeks post-operatively, headaches, dizziness and ataxia completely disappeared, and the patient regained his daily activity. His clinical improvement has been permanent at five years of follow-up.

\section{Discussion}

AC are benign developmental anomalies located within the arachnoid membrane, and mostly asymptomatic [3]. They are estimated to account for approximately $1 \%$ of all intracranial mass lesions [4]. Most AC are located in the middle cranial fossa [1]. Only $5 \%$ to $10 \%$ of the cysts have been found to occur in the posterior cranial fossa [5]. There is no age predilection and the male to female ratio is 3:1 [5]. The hallmark of a posterior fossa AC is the variability in presenting signs and symptoms [6] [7]. Posterior fossa AC are often unexpected, because they remain asymptomatic or can cause vague and non-specific symptoms such as headache, dizziness, hearing loss, tinnitus, lower cranial nerve palsies, facial numbness, cerebellar and pyramidal signs, psychomotor retardation, seizures, and symptoms compatible with multiple sclerosis [7]. No single symptom or group of symptoms defines posterior fossa arachnoid cysts. Hence, these lesions should be considered as a potential diagnosis when the symptoms are longstanding, vague, or difficult to explain [8]. The etiology of AC remains controversial; it is presumably congenital, and the cyst is supposed to result from splitting of the arachnoid membrane. However, acquired etiologies, such as inflammation and trauma have also been proposed [9]. Several mechanisms could be responsible for gradual enlargement of an AC. Intracystic hemorrhage, an osmotic gradient allowing a passive fluid-diffusion into the cyst, a ball-valve mechanism, or an active secretion from the cyst wall is the main theories to explain cyst growth [2]. Magnetic resonance imaging (MRI) is the preferred method of investigation. Signal intensity is identical to that of CSF. MRI assists in differentiating between other cystic lesions of the posterior fossa. In fact, AC can be differentiated from an epidermoid cyst by the abnormal higher signal intensity of the latter on diffusion-weighted and FLAIR imaging sequences.

The differential diagnoses also include Dandy-Walker malformation, its variant, mega cisterna magna [1] [10], and cerebellar hydatid cyst particularly in 
our context.

The key features in making the distinction between these possibilities are the position of the fourth ventricle, the tentorium, the torcula and its sinuses, the size of the posterior fossa, the presence of a vermis, and a communication between the cyst and the fourth ventricle [11]. In our case, since the vermis were present and normal position of the torcula were found, the cyst did not communicate with the fourth ventricle which was rostrally deviated, our preoperative diagnoses were arachnoid versus hydatid cysts of the posterior fossa. At surgery, the macroscopic aspect was in favor an AC. Various procedures of surgical approach for arachnoid cyst have been reported [1] [2] [11]: Cyst fenestration, stereotactic punctures, endoscopic cyst fenestration, cystoperitoneals shunt, cyst marsupialization into the subarachnoid space, and complete or partial resection of the cyst wall via a direct surgical approach. In our context, we believe that the direct approach is the surgical procedure of choice since hydatid cyst can have similar radiologic characteristics and hydatidosis still rages in the endemic states in our country. Besides, direct surgical approach allows complete resection of the arachnoid wall and communication of the cyst content with the subarachnoid spaces in order to prevent recurrences and the anatomic location of the lesion was amenable to complete resection.

The clinical outcome of our patient is remarkably good at 5 years follow up. In 2015 Wang and al studied the clinical outcome of surgical treatment for symptomatic arachnoid cysts in adults. They found that posterior fossa AC had good clinical outcome, in line with our results and that dizziness and vomiting were predictors of favorable outcome [12]. A recent review of the effectiveness of surgical intervention for symptomatic intracranial arachnoid cyst in adults showed that surgical intervention is an effective approach to eliminate symptoms caused by posterior fossa AC [13].

\section{Conclusion}

Arachnoid cysts are benign cysts occurring usually in the supratentorial space and containing cerebrospinal fluid. Their occurrence in the posterior fossa is rare. Magnetic resonance imaging is the exam of choice. Complete cyst resection is the most reliable option for prevention of recurrence. Long-term follow-up shows improvement of the majority of the preoperative symptoms.

\section{Patient Consent}

An informed written consent was obtained from the patient.

\section{Conflicts of Interest}

The authors declare no conflicts of interest regarding the publication of this paper.

\section{References}

[1] Daneyemez, M. and Gezen, F. (1999) Presentation and Management of Supraten- 
torial and Infratentorial Arachnoid Cysts. Journal of Neurosurgical Sciences, 43, 115-123.

[2] Samii, M., Carvalho, G.A., Schuhmann, M.U. and Matthies, C. (1999) Arachnoid Cysts of the Posterior Fossa. Surgical Neurology, 51, 376-382.

https://doi.org/10.1016/S0090-3019(98)00095-0

[3] Lockhat, F., Mubaiwa, L., Clarke, B. and Corr, P. (2005) Imaging of a Fourth Ventricle Arachnoid Cyst in a Child. Clinical Radiology Extra, 60, 35-37. https://doi.org/10.1016/j.cradex.2004.11.003

[4] Tsuboi, Y. (2005) Huge Arachnoid Cyst in the Posterior Fossa: Controversial Discussion for Selection of the Surgical Approach. Childs Nervous System, 21, 259-261. https://doi.org/10.1007/s00381-004-1006-3

[5] Osborn, A.G. (1994) Miscellaneous Tumors, Cysts, and Metastases. In: Osborn, A.G., Ed., Diagnostic Neuroradiology, Mosby, St. Louis, MO, 39-42.

[6] Haberkamp, T.J., Mansell, E.M., House, W.F., Levine, S. and Piazza, L. (1990) Diagnosis and Treatment of Arachnoid Cysts of the Posterior Fossa. Otolaryngology-Head and Neck Surgery, 103, 610-614.

https://doi.org/10.1177/019459989010300414

[7] O’Reilly, R.C. and Hallinan, E.K. (2003) Posterior Fossa Arachnoid Cysts Can Mimic Meniere’s Disease. American Journal of Otolaryngology, 24, 420-454. https://doi.org/10.1016/S0196-0709(03)00097-8

[8] Thinakara-Rajan, T., Janjua, A. and Srinivasan, V. (2006) Posterior Fossa Arachnoid Cyst Presenting with Isolated Sensorineural Hearing Loss. The Journal of Laryngology \& Otology, 120, 979-982. https://doi.org/10.1017/S0022215106002337

[9] Joong-Uhn, C. and Dong-Seok, K. (1998) Pathogenesis of Arachnoid Cyst: Congenital or Traumatic? Pediatric Neurosurgery, 29, 260-266.

https://doi.org/10.1159/000028733

[10] Kollias, S.S., Ball Jr., W.S. and Prenger, E.C. (1993) Cystic Malformations of the Posterior Fossa: Differential Diagnosis Clarified through Embryologic Analysis. RadioGraphics, 13, 1211-1231. https://doi.org/10.1148/radiographics.13.6.8031352

[11] Arai, H. and Sato, K. (1991) Posterior Fossa Cysts: Clinical, Neuroradiological and Surgical Features. Child s Nervous System, 7, 156-164.

https://doi.org/10.1007/BF00776713

[12] Wang, Y.Q., Wang, F., Yu, M.K. and Wang, W.P. (2015) Clinical and Radiological Outcomes of Surgical Treatment for Symptomatic Arachnoid Cysts in Adults. Journal of Clinical Neurosciences, 22, 1456-1461. https://doi.org/10.1016/j.jocn.2015.03.016

[13] Hayes, M., TerMaath, S.C., Russel Crook, T. and Killefer, J.A. (2019) A Review on the Effectiveness of Surgical Intervention for Symptomatic Intracranial Ararchnoid Cysts in Adults. World Neurosurgery, 123, 259-272.

https://doi.org/10.1016/j.wneu.2018.11.149 